\title{
Development and validation manual education for patients submitted to chemotherapy
}

\begin{abstract}
Objective: To describe the process of construction and validation of a manual of guidelines for cancer care to patients undergoing chemotherapy.

Method: Descriptive research carried out from 2016 to 2017 from the stages: situational diagnosis; collection of information; summary and selection of content; text elaboration; creation of illustrations; consultation with specialists; public consultation with target group; adequacy of the manual; and evaluation of the Flesch Readability Index (FLI). The Delph Technique was used for the validation process, which was performed by 10 experts in the thematic area of the manual. The concordance index of at least $80 \%$ was considered to guarantee the validation of the material.
\end{abstract}

Results: the items covered in the manual evaluation instrument were divided into three blocks: objectives, structure and presentation, and relevance that obtained the Concordance Index (CI) of 93.75\%.

Conclusion: the manual was considered valid for content and appearance. Patients undergoing chemotherapy and without professional follow-up and information can make them more sufferers, which contradicts the philosophy of palliative care to prevent human suffering in all dimensions of care, including spiritual, psychosocial.

Keywords: oncology nursing, nursing care, validation studies, chemotherapy
Volume 2 Issue $6-2018$

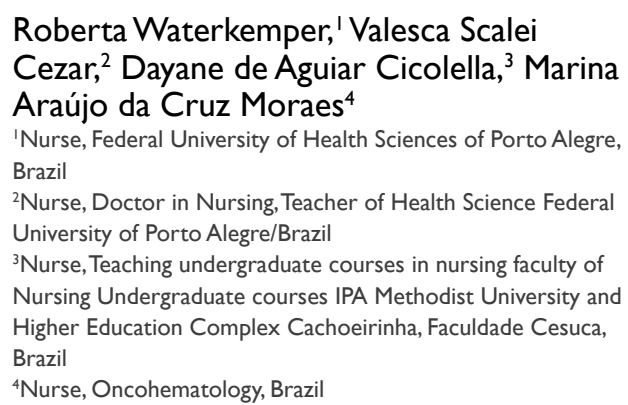

Correspondence: Roberta Waterkemper, Nurse, $\mathrm{PhD}$ in Nursing,Adjunct III, Federal University of Health Sciences of Porto Alegre -UFCSPA, Brazil, Email robsvater@gmail.com

Received: November 10, 2018 | Published: December 14, 2018

\section{Introduction}

Cancer is one of the world's chronic non-communicable chronic diseases (CNCD) and one of the five diseases with the highest mortality rate. Only in the biennium 2018-2019, in Brazil, it is expected that 600 thousand new cases of cancer will occur per year. ${ }^{1}$ From this scenario, public policies highlight the need to develop strategies for disease prevention and control, as well as access to diagnosis and initiation of treatment within 60 days, as well as a safe care that minimizes the undesirable effects of therapeutic treatment. ${ }^{2,3}$ In Brazil, the law No. $12,732 / 12$ was published in 2012 , which established the obligation for health services to initiate cancer treatment within 60 days after diagnosis, created mainly under the impact of the high initial staging, already indicating the need for a look of palliative care and morbidity. Cancer mortality is increasingly emphasized in the early diagnosis to increase the capacity for control, cure and prevention of suffering from illness ${ }^{4,5}$ and in this perspective, the American Society of Clinical Oncology (ASCO), the European Society of Medical Oncology and the National Network of Comprehensive Cancer advocate the integration of palliative care in clinical oncology. It is understood that this early fusion, from the moment of diagnosis, especially in the most advanced cases of cancer, can improve the quality of life and care. ${ }^{6}$

By having as one of its principles to prevent suffering from diagnosis health services need to adopt this philosophy throughout the therapeutic itinerary of patients and families. Suffering involving falling ill from cancer is no longer a novelty and people somehow already know some of the treatments. Among them, and most common, performed for healing and relief of signs and symptoms is chemotherapy or "chemiluminescence" term used in the communication of patients. This isolated treatment modality or in combination with other drugs brings toxicities that, if not managed, can make treatment a process that generates anxiety, fears and mild physiological reactions to severe. ${ }^{5-7}$
The lack of communication and guidance by health professionals, especially before the start of chemotherapy, has been pointed out in the literature as one of the contributing factors for non adherence to self-care guidelines and increased complaints and evidence of toxicity being controlled. ${ }^{6,7}$ Second, an American Society of Clinical Oncology (ASCO) is rescued during treatment, during and after chemotherapy with effective communication. This is represented by information recording in physical and computerized practice and an education for self-care. Communication can be done verbal rather than verbally. The most common books are: books, guides and work on educational health technologies. These technologies play an important role in communicating with patients about the instructions given by the nurse to be an accessible resource that can facilitate self-care, especially in the home environment. ${ }^{8}$ Most often, it is at home that patients and their families experience the toxicities of treatment, including the most serious ones. ${ }^{8} 9$

To ensure the effectiveness of educational materials whether in the form of manuals, brochures, care and management protocols need to be tested, evaluated and validados..$^{10}$ This study aims to describe the process of elaboration and validation of an educational manual for cancer patients undergoing chemotherapy ambulatory. It is believed that this manual will enable the patient and family to know and recognize common reactions physiological, psychological and initiate self-care actions that can minimize or prevent its aggravation and unnecessary suffering.

\section{Method}

It is a methodological development study which has as main objectives to guide the elaboration of a method of research, organization and data analysis for its development, validation and evaluation of tools and research techniques. ${ }^{10}$ It was conducted between 2016 to 2017 at a referral Hospital for prevention, diagnosis 
and treatment of cancer in southern Brazil. The study population consisted of patients diagnosed with cancer in outpatient care and health professionals working in oncology. The information for the elaboration of the manual was organized into six steps: situational diagnosis; collection of information; selection and summary of the contents; text elaboration; creation of illustrations; validation. To step situational diagnosis, informal talks were held with all professionals involved in the care of patients in outpatient chemotherapy, and the analysis of information in electronic medical records. Based on the information obtained in this stage, a literature review was performed on the main toxicities presented by patients in chemotherapy and related nursing care, from online databases (Scielo, Medline and LILACS) without time limit, articles available online and full manuals of Health Ministry in Brasil and reference institutions in the area in Portuguese, English and Spanish. Criteria for content selection were texts addressing human studies, to describe the effects of chemotherapy and / or care provided by nurses was used as descriptors in Health Sciences (DECS) and keywords in Portuguese, English and Spanish, respectively: nursing (Nursing), chemotherapy (Drug therapy/Chemotherapy/, complications (Complications/ complicaciones), chemotherapy toxicity (toxicity of chemotherapy/ her toxicity of chemotherapy). Nausea (nausea/nausea), vomiting (vomiting/vomiting), diarrhea (diarrhea/diarrhea), fever (fever/ fiebre), fatigue (fatigue/tiredness), alopecia (alopecia/alopecia), mucositis (mucositis/mucositis), bleeding (hemorrhage/bleeding), anorexia (anorexia/anorexia),

The information obtained by this search associated with professional health and electronic medical records made it possible to gather and select relevant information, wrights the abstract and text. Text content was validated by Delph technique with a panel of 10 professional experts (nurse, doctor, physiotherapist, speech therapist, nutritionist) in oncology. It is a method of deduction and refining opinions of a group of experts to reach a group consensus through questionnaires with controlled feedback of opinions. Its main characteristics: anonymity, interaction, feedback and controlled the statistical grouping of the group's responses. ${ }^{11} \mathrm{We}$ obtained convergent expert opinions, known as consensus group setting a content validity index (CVI) to indicate the consistency of the views (Figure 1). ${ }^{10,12}$

The formal invitation to the participants occurred with the sending of an invitation letter. This was delivered personally by the researchers and, upon acceptance, the instrument of evaluation, the Informed Consent Form (TCLE) and a copy of the educational manual were delivered printed in an envelope. The technique was based on an evaluation instrument, adapted from a pre-existing questionnaire, after authorization by the author, with appropriate modifications in relation to the theme that was addressed in the educational manual of this study. Each item of the manual was evaluated according to the criteria of relevance (significance for the quality of nursing care); clarity (intelligibility, with objective and unambiguous expressions); relevance (reproduces what is recommended by protocols, guidelines and literature reviews) and simplicity (expresses a single idea, with no possibility of other interpretations). At the end of each block, the experts could justify their answers and / or make suggestions regarding the educational manual. The answers to the questions were presented in scale Likert ${ }^{13}$ type, ranging from 1- Inadequate to 4-Totally Adequate.

For the evaluation of the Content Validity Index (CVI), a value greater than or equal to 0.78 was considered. This index measures the proportion of judges who are in agreement on certain aspects of the instrument and its items. As the manual was evaluated by more than six specialists, the literature recommends a cut off point above $0.78 .^{13,14}$ Thus, for the full evaluation of the manual, the sum of all the calculated IVC was calculated using the sum of the values assigned by the likert scale by item and judge, divided by the number of judges and the maximum value attributed according to the data were tabulated, processed and analyzed using descriptive statistics.

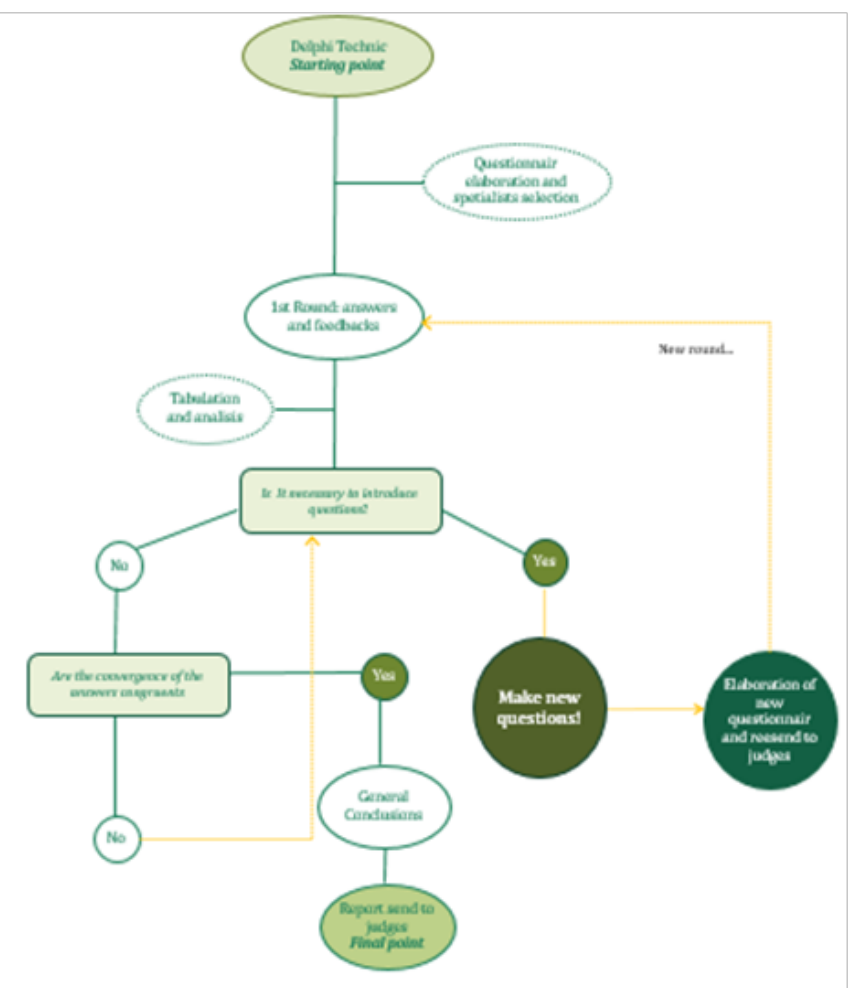

Figure I Development flowchart of the Delph Technique. Porto Alegre, 2018. Font: Waterkemper R. Production based on the delphy technic description references. Porto Alegre, 2018.

In addition, the percentage of at least $80 \%$ of responses assigned as adequate (A) and fully adequate (AT) for validation was considered. In this way, the A and TA responses of the judges should reach at least $80 \%$ to ensure validation. The item whose percentage was below $80 \%$ of agreement was analyzed and adjusted according to the suggestions of the specialists and recommendations of the literature based on clinical evidence. Data were tabulated, processed and analyzed using simple descriptive statistics.

After validation, the manual was presented to the institution to be used in the nursing consultations of the chemotherapy outpatient clinic and educational actions were carried out to prevent and treat adverse reactions related to treatment. The research subproject followed Resolution 466/12 15 of the National Health Council, being submitted to the Ethics and Research Committees (CEP) of the Irmandade Santa Casa de Misericórdia of Porto Alegre/ISCMPA and approved under protocol no. $1,476,850$.

\section{Results}

Study group consisted of 10 health professionals (five nurses, two physicians, a nutritionist, a physiotherapist and a speech therapist), all female. The average age of the referees was 36 years. The training time ranged from 2 to 35 years and the performance in the area from 
2 to 25 years. Regarding the qualification of health professionals, who were considered experts, after analyzing the Lattes Curriculum and according to the criteria of the expert scoring system used in the present study, one possessed the title of doctor and nine of specialists (Table 1).

Table 1 presents the answers given by the experts and the Concordance Variation Index (CVI) of each item of the evaluation. The evaluation of the validation process was analyzed through simple descriptive statistics from the experts' answers $(n=10)$ to the items: objective, structure and presentation and relevance of the evaluation instrument. Only two rounds were carried out: the first evaluation with change and resubmission for the second round and the submission of the report. In the first round of the Delphi technique, after the literature review to compose the manual, the educational material constructed to begin the validation was sent to the judges. This was done from the instrument containing questions with Likert type scale evaluation and at the end of each block a space for placement of suggestions/corrections. In this first round few changes were suggested. The items that had partially adequate evaluation were: coherence of the information, adequacy to the objectives of the institution and coherence between the language and the public. At the end of each block, the experts justified their responses and/or provided suggestions where appropriate. For the second round, the contributions of the first one were analyzed and the necessary changes were made which were considered pertinent considering that the IVC was around $78 \%$.

Table I The structure of the manual - focus for a dialogical text

\begin{tabular}{|c|c|c|c|c|c|c|c|c|c|c|c|c|c|}
\hline \multirow[t]{2}{*}{ Rated items } & \multicolumn{13}{|c|}{ Judges } \\
\hline & اנ & $\mathbf{J 2}$ & J3 & J4 & J5 & J6 & J7 & J8 & J9 & J10 & IVC* & MB \pm & MG§ \\
\hline \multicolumn{14}{|l|}{ Goals } \\
\hline $\begin{array}{l}\text { The information is consistent with the needs of patients undergoing } \\
\text { chemotherapy }\end{array}$ & PA & A & A & A & TA & TA & TA & TA & TA & TA & 0,87 & 0,89 & 0,89 \\
\hline The information is consistent to achieve a health education process; & A & A & A & A & A & TA & TA & TA & TA & TA & 0,87 & & \\
\hline Information can contribute to the process of behavior change and attitude; & A & A & TA & TA & TA & TA & TA & TA & TA & TA & 0,95 & & \\
\hline $\begin{array}{l}\text { The information meets the objectives of the institution and other institutions } \\
\text { working with chemotherapy treatment; }\end{array}$ & PA & A & A & A & TA & TA & TA & TA & TA & TA & 0,87 & & \\
\hline \multicolumn{14}{|l|}{ Structure and presentation } \\
\hline $\begin{array}{l}\text { The manual is appropriate for guidance to patients undergoing } \\
\text { chemotherapy; }\end{array}$ & A & A & A & TA & TA & TA & TA & TA & TA & TA & 0,92 & 0,89 & \\
\hline The information is presented in a clear and objective way; & PA & A & A & TA & TA & TA & TA & TA & TA & TA & 0,87 & & \\
\hline $\begin{array}{l}\text { The information presented is scientifically adequate to national and } \\
\text { international references; }\end{array}$ & A & A & A & TA & TA & TA & TA & TA & TA & TA & 0,92 & & \\
\hline $\begin{array}{l}\text { The material is appropriate to the socio-cultural level of the proposed } \\
\text { target audience; }\end{array}$ & PA & A & A & A & TA & TA & TA & TA & TA & TA & 0,87 & & \\
\hline There is a logical sequence of proposed content; & A & A & A & TA & TA & TA & TA & TA & TA & TA & 0,92 & & \\
\hline The information is well structured in agreement and spelling; & PA & A & A & A & TA & TA & TA & TA & TA & TA & 0,87 & & \\
\hline $\begin{array}{l}\text { Cover, back cover, acknowledgment and / or presentation information is } \\
\text { consistent with the purpose of a patient / family / caregiver guidance manual; }\end{array}$ & A & $A$ & A & TA & TA & TA & TA & TA & TA & TA & 0,92 & & \\
\hline The illustrations are expressive and sufficient; & PA & A & A & A & TA & TA & TA & TA & TA & TA & 0,87 & & \\
\hline The number of pages is adequate; & $A$ & A & $A$ & A & $A$ & TA & TA & TA & TA & TA & 0,87 & & \\
\hline \multicolumn{14}{|l|}{ Relevance } \\
\hline $\begin{array}{l}\text { The topics cover aspects related to prevention and control / treatment } \\
\text { / management of signs, symptoms and toxicity reactions that should be } \\
\text { reinforced; }\end{array}$ & PA & PA & A & A & TA & TA & TA & TA & TA & TA & 0,85 & 0,91 & \\
\hline $\begin{array}{l}\text { The manual proposes to the patient to acquire knowledge to perform self- } \\
\text { care, as well as to family and / or caregivers; }\end{array}$ & PA & TA & TA & TA & TA & TA & TA & TA & TA & TA & 0,95 & & \\
\hline It is suitable for use by any healthcare professional; & PA & $A$ & TA & TA & TA & TA & TA & TA & TA & TA & 0,92 & & \\
\hline
\end{tabular}

After this return of the second round were made changes related to textual coherence and language. In the general evaluation of the items by the judges in the second round, the IVC was above $80 \%$, obtaining a general average of $89 \%$. In relation to the objectives and structure and presentation, the IVC was $89 \%$. On the other hand, relevance was 91\% (Table 1).

From the situational diagnosis and review of the literature it was verified that among the main toxicities that would need to be addressed in the manual were: Nausea and vomiting, diarrhea, fever, fatigue, alopecia, mucositis, bleeding, anorexia, constipation and hyperpigmentation of the skin. In textual elaboration, the authors sought to organize information in order to depict the main toxicities presented by the patient undergoing chemotherapy. Some suggestions were not fully inserted in the manual after reviewing scientific evidence for not being standardized by the institution, even though they were considered strong recommendations, requiring additional studies for its feasibility in the context of the study.

Thus, the guide is composed of four main topics as can be seen in the Table 2 below: 
Table 2 Main topics that make up the guidance manual Porto Alegre, 2018.

Information that make up the manual

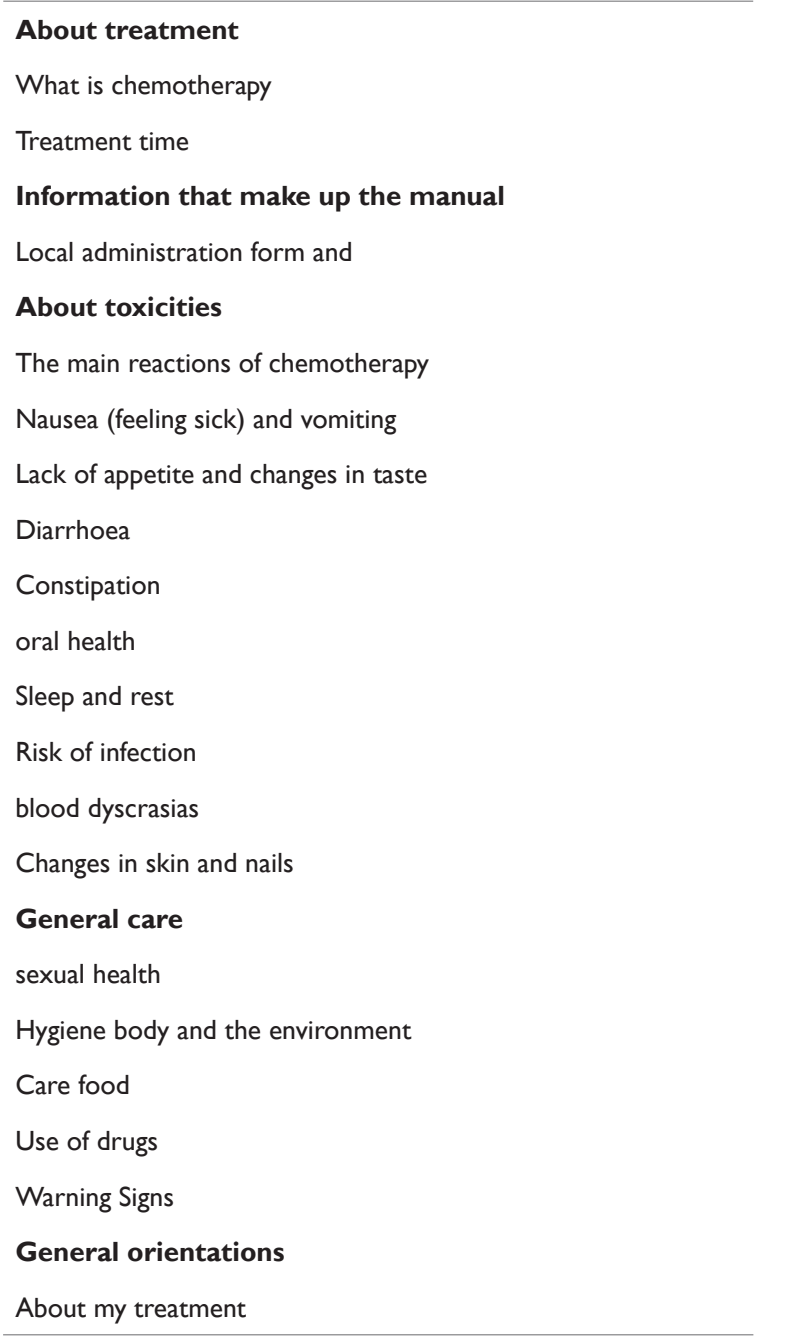

Considering the context of illness of cancer patients, the effects of treatment on the biopsychosocial dimensions and health education, textual construction of the manual was organized having as axis titles as a question. This idea aimed to stimulate the reader (patient/family) to seek the answer in the text, from common questions that occur between patients and family members as can be seen in the summary (Figure 2).

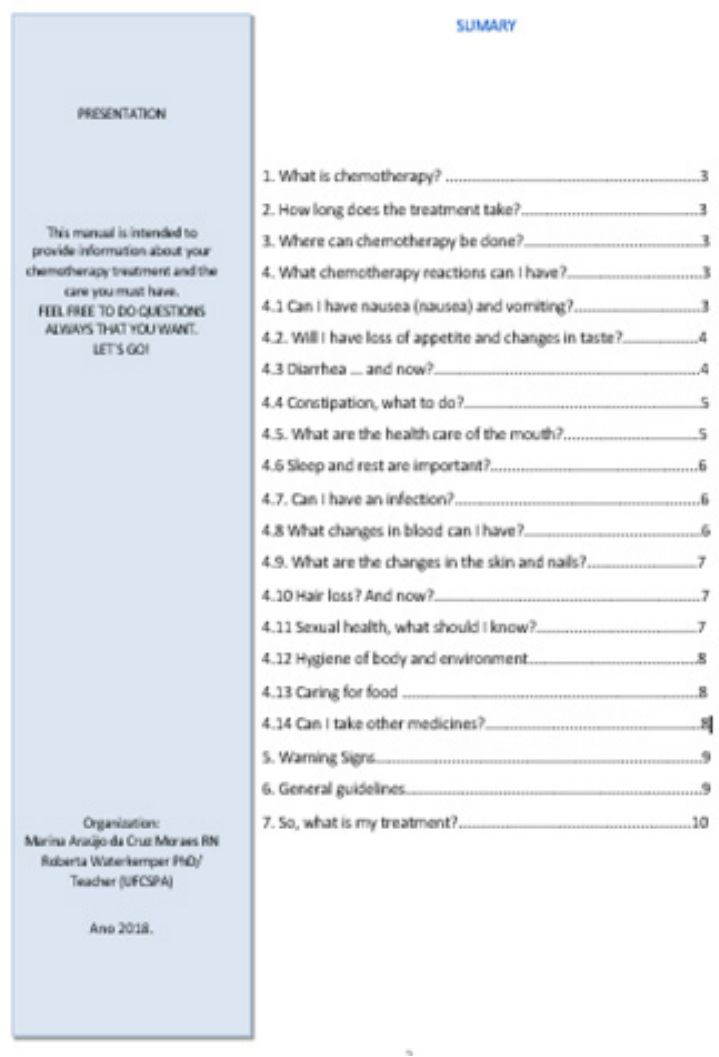

Figure 2 Summary of representation in the form of questions. Porto Alegre, 2018

Finally, the guide is composed of 10 pages and one illustration. The manual aims to facilitate the process of orientation that nurses need to perform the first nursing consultation before the start of the outpatient treatment. Figure 3 shows the proposed layout of the cover, abstract, one of the topics and final sheet containing information related to the chemotherapy protocol, contact phone and possible reactions related to specific protocol.

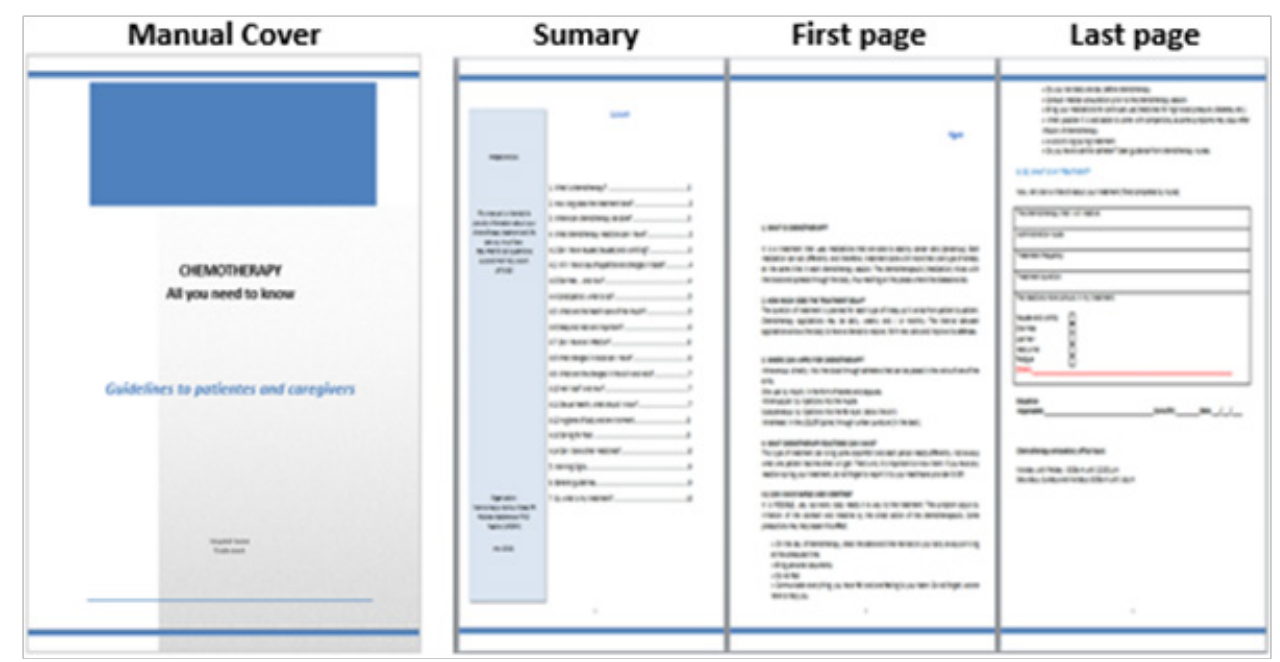

Figure 3 Proposal diagramming cover and organization of the manual's topics. 


\section{Discussion}

The diversity of professional experts was a favorable factor regarding process validation, as they grouped different expertise in the subject addressed by the manual, resulting in a multidisciplinary work. The biopsychosocial overview is critical in the treatment of cancer patients, given the complexity of therapeutic modalities and possible acute complications that can lead. ${ }^{14}$

The number of rounds of the Delphi technique varies according to some references and is generally recommended above two when necessary. In this study two rounds were performed that reached a significant level of convergence. The strength of the judges' overall assessment of the items was the relevance of the material to a $91 \%$ IVC and "appropriate and fully adequate" concept. It is understood that the material analyzed is suitable for use in clinical practice, which does not preclude a more thorough evaluation. ${ }^{15}$

Thus, observing the legal aspects of the nurse's role in chemotherapy ${ }^{16,17}$ the health promotion and the prevention of toxicities and adverse effects through the education of patients and their families is highlighted as their competence. This process should be carried out by means of the elaboration and implementation of educational manuals adapted to their social reality, which provide specialized information and enable humanized care in the environment in which patients undergo chemotherapy. However, it is noteworthy that this part of the service is the creation of spaces in which patients and family members can seek information and validate their understanding, mainly about the side effects and the resources of support for the attendance of health needs that may arise along of treatment as psychosocial, guidance of prosthetic advice and additional care.

In this sense, it is understood that the developed manual presents an accessible language and can be used to educate patients and families about chemotherapy treatment. However, it is worth mentioning that the educational manual does not replace the verbal guidance provided by the nurse during the nursing consultation, although it is of great value for strengthening the recommendations. ${ }^{13,14}$

This guidelines manual may contribute to the relief of side effects through self-care and even prevention when initiated early and in a qualified manner. When patients know about their diagnosis and treatment, they initially experience anxiety and, for this reason, they may easily have difficulties or even become unable to understand or apprehend information related to routine chemotherapy and toxicities. ${ }^{17}$

Thus, the education of patients and families by qualified nurses and with validated support material throughout the therapy has evidenced empowering patients and their families for self-care and, reducing the demand for health services for their management and improving anxiety levels the quality of life and care. ${ }^{17}$

It is known that health education can be mediated by a variety of technologies, from printed materials to applications for mobile phones and it is important to understand that these support devices used help the patient to adopt or modify behaviors that allow the attendance of their needs. The printed material presents a good alternative as the cost-benefit is attractive, and it becomes easy to distribute, enabling the dissemination of knowledge among patients and families, facilitating the care process. ${ }^{13,14}$
The study carried out has limitations that can be revised and allow the development of new proposals. There was little involvement of the judges in the written suggestions and comments, as well as the delay in the return of the evaluation tool, which could better support changes in the material presented.

\section{Acknowledgments}

Irmandade Santa Casa de Misericórdia de Porto Alegre (ISCMPA), Serviço de Enfermagem da Quimioterapia (Sistema Único de Saúde/ SUS) and Universidade Federal de Ciências da Saúde de Porto Alegre (UFCSPA)

\section{Conflicts of interest}

The author declares there is no conflict of interest.

\section{References}

1. Health Ministry (BR). National Cancer Institute. Estimate 2018: cancer incidence in Brazil. Rio de Janeiro: Inca; 2017.

2. Andrade KBS, Francz ACL, Grellmann MS, et al. Nursing care: evaluation of adherence to self-care of patients undergoing radiotherapy. Magazine UERJ nursing. 2014;22(5):622-628.

3. Silva SYM, Lima FLT, O'dwyer G, et al. Cancer-care policy in Brazil after the creation of the single health system. Brazilian Journal of oncology. 2017;63(3):177-187.

4. Nishijima TF, Deal AM, Williams GR, et al. Chemotherapy treatment for toxicity risk score Decisions in older adults with advanced solid tumors. Oncologist. 2018;23(5):573-577.

5. Visentin A, Mantovani MF, Kalinke LP, et al. Palliative therapy in adults with cancer: a cross-sectional study. Rev Bras Enferm. 2018;71(2):272279 .

6. Swami M, Case AA. Effective palliative care: what is Involved? Oncology (Williston Park). 2018;32(4):180-184

7. Coolbrandt A, Wildiers H, Aertgeerts B, et al. Systematic development of chemo-support, a nursing intervention to support adult patients with cancer in dealing with chemotherapy-related symptoms at home. BMC Nurs. 2018;17:28.

8. American Society of Clinical Oncology (ASCO). Qopi ${ }^{\circledR}$ Certification program standards manual. Standards manual required processes and documentation to meet certification standards and elements. 2018.

9. Kav S, Johnson J, Rittenberg C, et al. Role of the nurse in patient education and follow-up of people receiving oral chemotherapy treatment: an international survey. Support care cancer. 2008;16(9):1075-1083.

10. Polit DF, Beck CT. Fundamentos da pesquisa em enfermagem: avaliação de evidências para a prática de enfermagem. 7th ed. Porto Alegre: Artmed; 2011

11. Sousa CS, Turrini RNT. Construct validation of educational technology for patients through the application of the delphi technique. Acta Paul Enferm. 2012;25(6):990-996.

12. Teles LMR, Oliveira AS, Campos SC, et al. Development and validating an educational booklet for childbirth companions. Rev Esc Enferm Usp. 2014;48(6):977-984.

13. Salles OS, Castro RCBR. Information material validation to patients undergoing chemotherapy and their familiares. Rev Esc Enferm Usp. 2010;44(1):182-189. 
14. Flávia CAM, Narley NPV, Manzi N, et al. Implementation of educational consultation manuals in nursing: opinion of Patients submitted to antineoplastic chemotherapy. Journal of nursing UFPE. 2017;11(5):1757-1762.

15. Brazil. Ministry of health. Resolution no. 466 of 12 December 2012.

16. Federal Nursing Council (COFEN). COFEN Resolution No. 569/2018.
Approves the technical regulation of the activities of nurses in cancer chemotherapy.

17. Jivraj N, Gallagher IT, Papadakos J, et al. Patients and empowering caregivers with knowledge: The development of the nurse-led Gynecologic Oncology chemotherapy education class. Canadian oncology nursing journal. 2018;28(1):4-7. 